\title{
Endoscopic necrosectomy under fluoroscopic guidance - a single center experience
}

\author{
Marian Smoczyński, Mateusz Jagielski, Anna Jabłońska, Krystian Adrych \\ Department of Gastroenterology and Hepatology, Medical University of Gdansk, Gdansk, Poland
}

Videosurgery Miniinv 2015; 10 (2): 237-243

DOI: $10.5114 /$ wiitm.2015.52058

\begin{abstract}
Introduction: Our report presents a technique of necrotic tissue removal during transmural drainage of walled-off pancreatic necrosis (WOPN) that is an alternative to the one that has already been described in the literature.

Aim: To assess the effectiveness and safety of endoscopic necrosectomy performed during transmural drainage of symptomatic WOPN.

Material and methods: Within the years 2012-2013, 64 patients underwent endoscopic treatment of symptomatic WOPN in our center. Eight patients underwent endoscopic necrosectomy during transmural drainage. Fragments of necrotic tissues were removed from the collection's cavity under fluoroscopic guidance using a Dormia basket. The results and complications of treatment were compared retrospectively.

Results: Sixty-four patients with WOPN underwent transmural drainage under endoscopic ultrasonography (EUS) guidance. Eight patients (12.5\%, 5 women and 3 men, mean age 57.25 years) were qualified for endoscopic necrosectomy. Transmural transgastric access was made in 7 patients and transduodenal access in 1 patient. Additional percutaneous drainage was used in 2 patients. Active drainage was continued for 24 days (11-44 days). The mean number of endoscopic procedures was 4.75 (3-9). The average number of necrosectomy procedures during drainage was 1.75 (1-4). Complications of endotherapy occurred in 2/8 (25\%) patients, and they were not directly connected with necrosectomy. Therapeutic success after the end of active drainage was achieved in all patients. During a 6-month follow-up no recurrence of the collection was observed.

Conclusions: Endoscopic necrosectomy under fluoroscopic guidance is an effective and safe method of minimally invasive treatment in a selected group of patients with symptomatic WOPN.
\end{abstract}

Key words: endoscopic necrosectomy, endoscopic drainage, transmural drainage, walled-off pancreatic necrosis, acute pancreatitis.

\section{Introduction}

During the last 20 years the strategy of treatment of necrotizing acute pancreatitis has changed. Technological progress has led to the development of minimally invasive techniques to treat walledoff pancreatic necrosis (WOPN). Minimally invasive methods include procedures performed with an endoscope, laparoscope or nephroscope that enable transperitoneal, retroperitoneal, transmural or transpapillary access to the necrosis [1].

Endoscopic drainage/debridement as a minimally invasive technique is an effective and safe method of treatment in patients with symptomatic WOPN and should be one of the first therapeutic techniques in a selected group of patients [2]. Despite its high effectiveness, endoscopic drainage also has some limitations including lower ability to remove

\section{Address for correspondence}

Mateusz Jagielski MD, Department of Gastroenterology and Hepatology, Medical University of Gdansk, 17 Smoluchowskiego St,

80-214 Gdansk, Poland, phone: +48 5834936 59, e-mail: matjagiel@gmail.com 
poorly liquefied necrosis [3]. Patients without clinical improvement despite the drainage require mechanical removal of tissue fragments from the collection's cavity. Endoscopic necrosectomy increases the effectiveness of drainage [4], but it is a technically difficult procedure connected with a high risk of complications.

In our report we want to present an analysis of our own results of treatment in patients with symptomatic WOPN during 2 years (2012-2013). In our center since 2001 we have used transmural (transgastric or transduodenal) access to pancreatic necrosis that is often accompanied by percutaneous access. In a selected group of patients we perform endoscopic necrosectomy.

Our study presents the technique of endoscopic necrosectomy consisting in the removal of necrotic tissues under fluoroscopic guidance without the need to insert a fiberoscope into the cavity of the WOPN. The results of treatment and the occurrence of complications were compared retrospectively.

\section{Aim}

The aim of the study was to assess the effectiveness and safety of endoscopic necrosectomy per- formed during transmural drainage in patients with symptomatic WOPN.

\section{Material and methods}

The study was approved by the Ethics Committee. All patients gave their informed consent for endoscopic procedures.

Between 2012 and 2013 in our center 64 patients underwent endoscopic treatment (Figure 1). The qualification for endotherapy was based on the presence of clinical symptoms connected with WOPN and the results of contrast-enhanced abdominal computed tomography (CECT) or abdominal magnetic resonance imaging (MRI). Endoscopic procedures were performed using duodenoscopes (Pentax ED3490TK and Pentax EG3870UTK, Japan). Gastropancreatic and duodenopancreatic fistulas were made under endoscopic ultrasonography (EUS) guidance with cystostome (Cystotome CST-10, Wilson-Cook, Ireland). A fluid sample from the collection was taken for microbial culture and to assess amylase activity. The morphology of the fluid aspired - dark brown color with visible fragments of necrotic tissues (debris) - was the basis of WOPN diagnosis. The stoma between the lumen

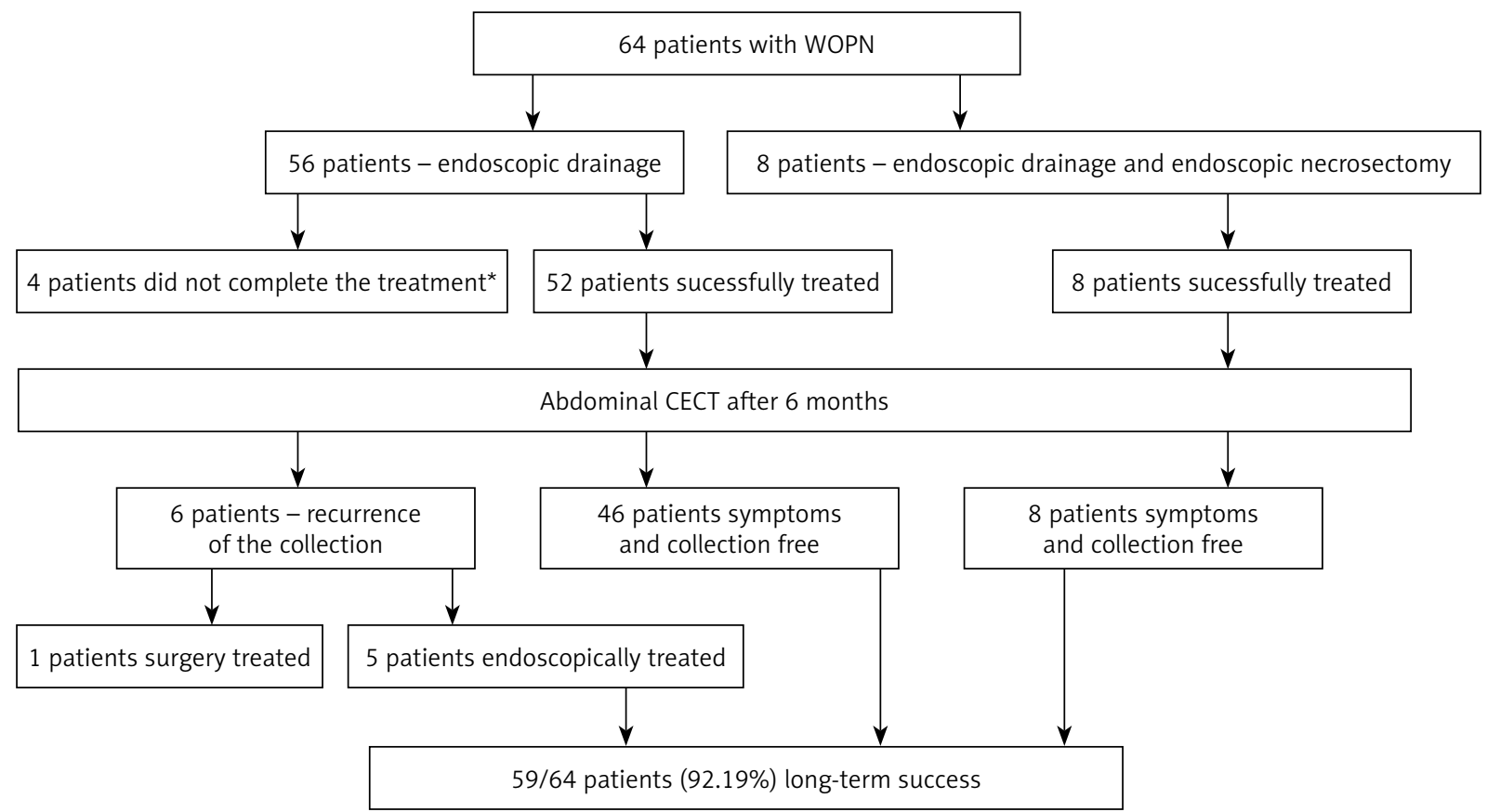

Figure 1. Study design ( ${ }^{\star} 1$ patient was treated surgically because of gastrointestinal perforation - during the operation the perforation was repaired and surgical drainage of WOPN was performed, 1 patient died during endotherapy because of splenic artery pseudoaneurysm hemorrhage, 2 patients - cessation of clinical symptoms but the collection size $>3 \mathrm{~cm}$ ) 


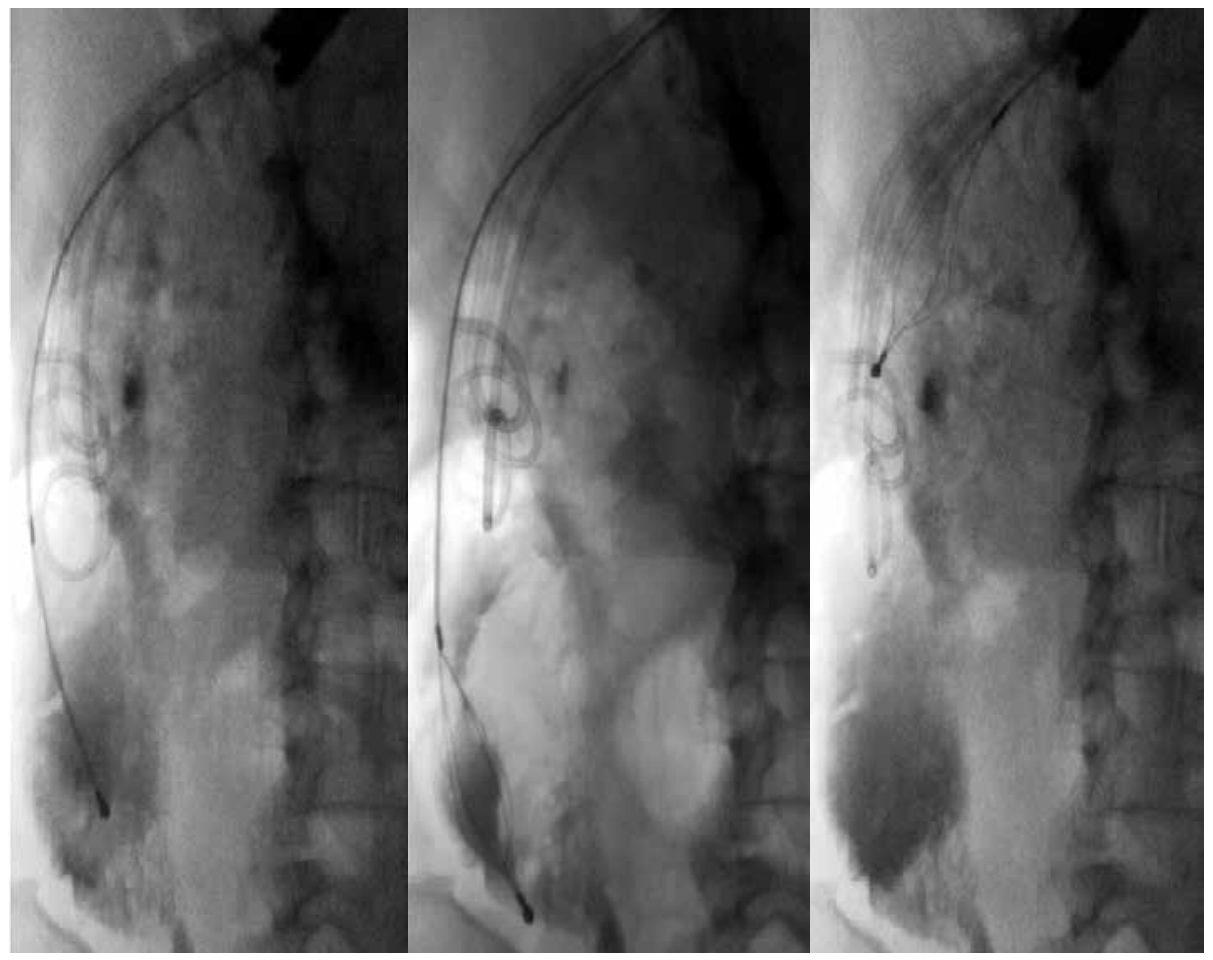

Photo 1. Endoscopic necrosectomy under fluoroscopic guidance. Dormia basket in the cavity of WOPN. Transmural stents also visible

of the gastrointestinal tract and the cavity of necrotic collection was widened using a high-pressure balloon $20 \mathrm{~mm}$ in diameter (Boston Scientific, USA). Through the stoma a $7 \mathrm{Fr}$ or $8 \mathrm{Fr}$ nasocystic drain (Wilson-Cook, Ireland) and a "double-pigtail" $7 \mathrm{Fr}$ or $10 \mathrm{Fr}$ stent (Wilson Cook, Ireland or Mar Flow, Switzerland) were inserted into the cavity of the collection. The necrotic collection was irrigated with saline solution $(200 \mathrm{ml})$ through a nasocystic drain every $2 \mathrm{~h}$ during the first $48 \mathrm{~h}$ and every $4 \mathrm{~h}$ in the subsequent days. Before the procedure all patients received antibiotics (ciprofloxacin or ceftriaxone with metronidazole). Routinely antibiotic therapy was continued for 2 weeks. In the case of clinical symptoms indicating infection of the collection antibiotic therapy was prolonged or microbial culture with antibiogram of fluid from the collection was repeated. In 8/64 (12.5\%) patients necrosectomy with a Dormia basket (FG-V422PR, Olympus, Japan) was performed under fluoroscopic guidance (Photos 1,2). The qualification criteria for endoscopic necrosectomy included lack of clinical improvement despite drainage, infection of the necrotic collection or a large amount of necrotic tissues observed during fluoroscopy (Photo 3) or EUS. Regression of the le-

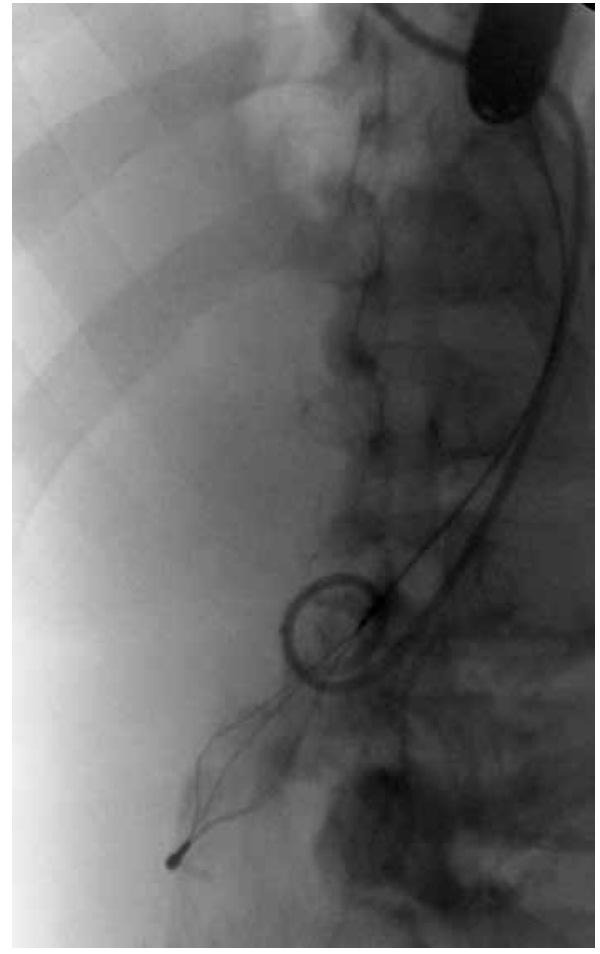

Photo 2. Endoscopic necrosectomy under fluoroscopy guidance. Dormia basket visible in the cavity of WOPN collection. The lower end of the nasal drain is also visible in the collection's cavity 


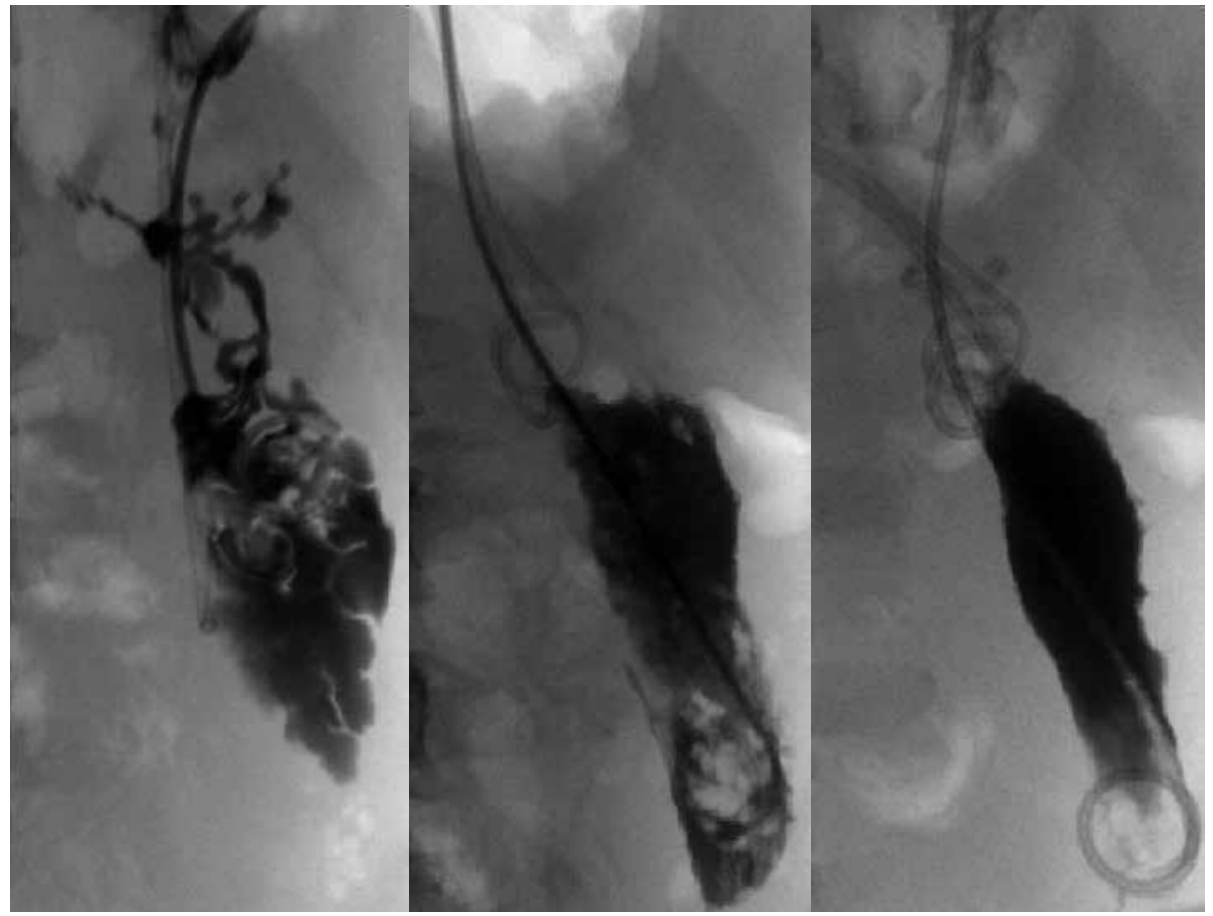

Photo 3. The contrast medium injected via the nasal drain fills the necrosis collection after the sequential necrosectomy procedures. Gradual regression of the collection is observed and there is a decrease in the amount of necrotic tissues visible during fluoroscopy

sions was assessed every 7 days on the basis of the clinical picture and abdominal ultrasonography (USG). Complete regression of the collection (diameter $<3 \mathrm{~cm}$ ) was diagnosed on the basis of abdominal CECT (Photos 4 A, B). Active drainage was discontinued in the case of symptom disappearance and the collection's diameter $<3 \mathrm{~cm}$. Therapeutic success was defined as the lack of clinical symptoms and the collection's size $<3 \mathrm{~cm}$ during a 6 -month follow-up from the end of active drainage.

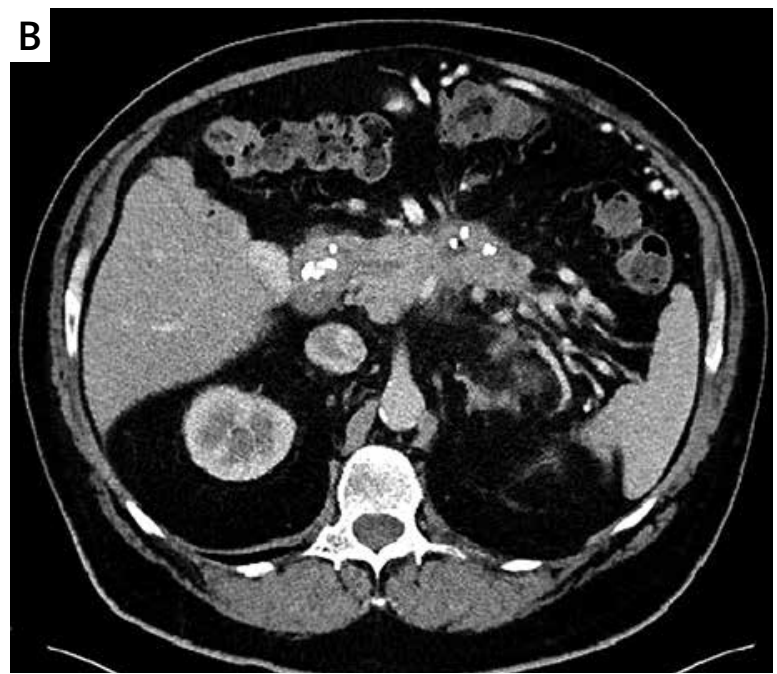

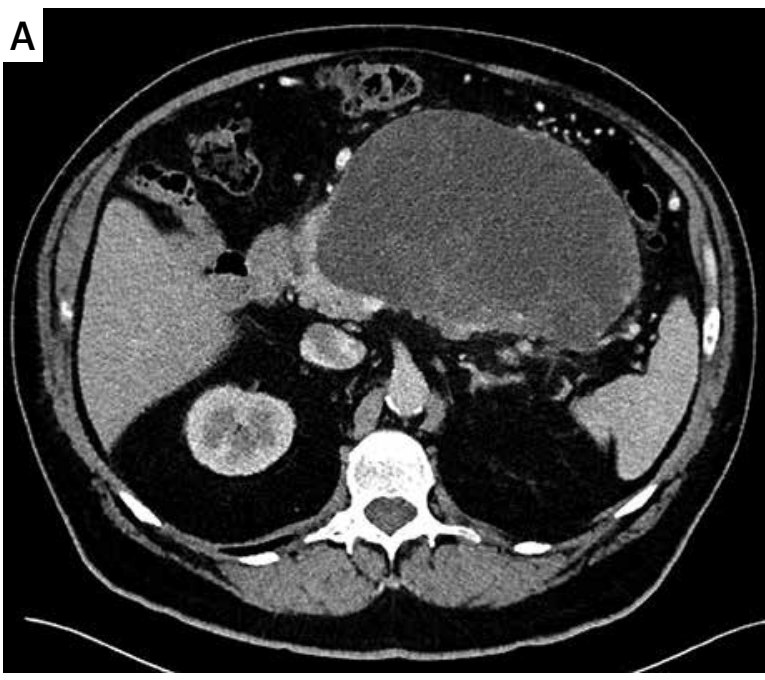

Photo 4 A, B. Contrast-enhanced abdominal computed tomography performed before and after endoscopic treatment of pancreatic necrosis. Active drainage was continued for 23 days and endoscopic necrosectomy was performed twice 
In the case of a main pancreatic duct leak observed during endoscopic retrograde pancreatography (ERP), sphincterotomy was performed (FlowCut KD-301Q0725 sphincterotome, Olympus, Japan) and a $7 \mathrm{Fr}$ pancreatic stent was placed in the main pancreatic duct (Wilson Cook, Ireland or Mar Flow, Switzerland).

\section{Results}

In the case of 8 patients ( 5 women and 3 men, mean age 57.25 years) indications for endoscopic necrosectomy were observed during transmural drainage (Photos 5 A, B). Therapeutic success was achieved in all patients. During a 6-month follow-up no recurrence of the collection occurred.

The etiology of acute pancreatitis was alcoholic in 3 patients and non-alcoholic in 5 patients ( 2 with gallstones, 1 iatrogenic, 2 idiopathic). The average time between acute pancreatitis and intervention was 14 weeks (6-22 weeks). The mean size of WOPN collection was $14.6 \mathrm{~cm}(10.6-22.0 \mathrm{~cm})$. In 5 patients mixed pancreatic necrosis was diagnosed (central and peripancreatic) and in 3 central pancreatic necrosis was observed.

The mean number of endoscopic procedures in 1 patient was 4.75 (3-9). The average duration of active drainage was 24 days (11-44 days). The mean number of necrosectomy procedures during the drainage in 1 patient was 1.75 (1-4). Transgastric access was used in 7 patients and transduodenal in 1 patient. Additional percutaneous drainage was conducted in 2 patients.

Complications of endotherapy occurred in $2 / 8$ (25\%) patients and were not directly connected with the necrosectomy procedure. In 1 patient perforation of the gastrointestinal tract was observed and was treated conservatively. Perforation occurred during fistulotomy formation. Dislocation of a transmural stent into the cavity of the WOPN during introduction of a nasal drain was observed in 1 patient. The stent was removed endoscopically using a Dormia basket.

Endoscopic retrograde pancreatography (ERP) was performed in 7/8 (87.5 \%) patients. In 1 patient with the collection localized in the pancreatic head it was not possible to contrast the main pancreatic duct. In 5/7 patients contrast medium leak from the main pancreatic duct was observed. In those patients a pancreatic stent was inserted in order to bridge the site of pancreatic duct injury. In 2/7 patients the main pancreatic duct was contrasted only in the pancreatic head (on the basis of abdominal CECT disconnected duct syndrome was diagnosed in those patients).

\section{Discussion}

Endoscopic therapy of WOPN has evolved in the space of recent years. In the first reports concerning endoscopic drainage/debridement of WOPN gastropancreatic or duodenopancreatic fistulas that were performed had a small diameter (10-12 mm) [5]. As the method was popularized the diameter of the fistula was increased up to $2 \mathrm{~cm}$ [6]. Dilation of the fistula to $20 \mathrm{~mm}$ enabled introduction of a fiberoscope into the WOPN cavity and performance of endoscopic necrosectomy [6]. Seewald et al. presented the results of treatment in 13 patients with pancreatic necrosis who had fragments of necrotic tissues
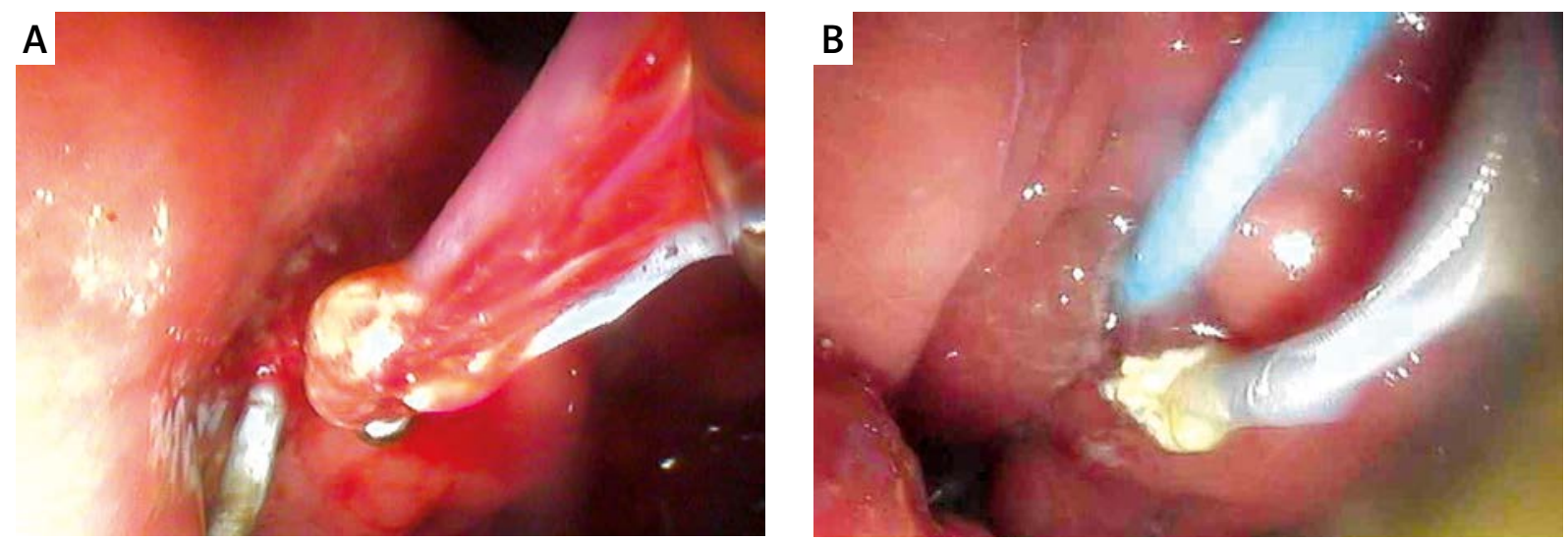

Photo 5 A, B. The Dormia basket with elements of necrotic tissues. Gastropancreatic fistula is visible with the nasal drain in its lumen inserted into the cavity of the necrosis collection 
removed using a Dormia basket after insertion of a gastroscope into the collection's cavity [6].

In our study we present the necrosectomy technique that is an alternative to the one described by many authors $[6,7]$. Therapeutic success was achieved in all patients $(8 / 8-100 \%)$ during a 6 -month follow-up. Complications of endoscopic therapy occurred in 2/8 (25\%) patients and were not directly connected with necrosectomy. The mean number of necrosectomy procedures in 1 patient was 1.75 (1-4), with an average of 4.75 (3-9) endoscopic procedures. Seewald et al. achieved therapeutic success in 11/13 (85\%) patients and complications occurred in $4 / 13$ (30.77\%) patients [6]. The number of necrosectomy procedures was $7(2-23)$ and of endoscopic lavage $12(2-41)$ [6]. Seifert et al. performed necrosectomy in 93 patients, achieving therapeutic success in 75 $(81 \%)$ patients, while complications were noted in $24(26 \%)$ patients, and the mean number of necrosectomy procedures was 6.2 (1-35) [7]. Papachristou et al. in a study performed on 53 patients obtained therapeutic success in $43(81 \%)$ patients; the mean number of procedures was 3 (1-12) [8]. Complications that were directly connected with the procedure were noted in $11(21 \%)$ patients [8]. In a multicenter study by Gardner et al. therapeutic success was achieved in 95/104 (91\%) patients, while complications occurred in $14 \%$ of patients [9]. A comparable index of success (19/22 patients; $86 \%)$ was achieved by Rische et al., with a complication rate of $13 \%$ [10]. In both studies the average number of endoscopic procedures was 4 $[9,10]$.

In a meta-analysis concerning endoscopic necrosectomy performed in 455 patients, van Brunschot et al. found that therapeutic success was achieved in $81 \%$ of patients, the mean number of procedures being $4(1-23)$ [11]. Complications occurred in $36 \%$ of patients. The most common complication was gastrointestinal bleeding, which occurred in $18 \%$ of patients. The second most common complication was perforation, noted in $4 \%$ of patients. In our study no case of gastrointestinal bleeding was noted, and perforation occurred in 1 patient.

Direct comparison of the study results is difficult not only because of the different number and variety of patients in study groups, but also because of the differences between treatment methods and aggressiveness of therapy used in the reports cited above. The duration of follow-up was also variable.
In most studies endoscopic necrosectomy is the basis of endoscopic treatment in patients with WOPN $[7,9]$. In our study the basis of the therapeutic strategy was endoscopic transmural drainage, and necrosectomy was performed in selected patients. We found indications for mechanical removal of necrotic tissues only in 8/64 (12.5\%) patients. Those were patients without clinical improvement despite the drainage who had a large amount of necrotic tissues visible during fluoroscopy and clinical symptoms suggestive of infection of the necrotic collection (abdominal pain, fever, elevated laboratory parameters of inflammation).

Jürgensen et al. published the results of treatment in patients who underwent endoscopic necrosectomy without active drainage as they only left transmural stents in the lumen of the fistula that provided passive drainage of the collection between the procedures [12]. According to our own experience, active drainage in patients with WOPN facilitates the removal of necrotic tissue fragments during endoscopic necrosectomy and is at the same time the basis of the therapeutic strategy in those patients.

The main limitations of our study are the small number of patients and a relatively short follow-up period. Nevertheless, the results confirm the effectiveness of this method as an alternative to other techniques used for treatment of patients after acute necrotic pancreatitis.

\section{Conclusions}

Endoscopic necrosectomy under fluoroscopic guidance during transmural drainage is an effective and safe method of treatment in patients with WOPN. In order to assess the utility of this technique more accurately, further studies conducted on a larger number of patients are needed.

\section{Conflict of interest}

The authors declare no conflict of interest.

\section{References}

1. Loveday BP, Mittal A, Phillips A, Windsor JA. Minimally invasive management of pancreatic abscess, pseudocyst, and necrosis: a systematic review of current guidelines. World I Surg 2008; 32: 2383-94.

2. Freeman ML, Werner J, van Santvoort HC, et al. Interventions for necrotizing pancreatitis: summary of a multidisciplinary consensus conference. Pancreas 2012; 41: 1176-94. 
3. Talreja JP, Kahaleh M. Endotherapy for pancreatic necrosis and abscess: endoscopic drainage and necrosectomy. J Hepatobiliary Pancreat Surg 2009; 16: 605-12.

4. Gardner TB, Chahal P, Papachristou GI, et al. A comparison of direct endoscopic necrosectomy with transmural endoscopic drainage for the treatment of walled-off pancreatic necrosis. Gastrointest Endosc 2009; 69: 1085-91.

5. Baron TH, Thaggard WG, Morgan DE, Stanley RJ. Endoscopic therapy for organized pancreatic necrosis. Gastroenterology 1996; 111: 755-64.

6. Seewald S, Groth S, Omar S, et al. Aggressive endoscopic therapy for pancreatic necrosis and pancreatic abscess: a new safe and effective treatment algorithm (videos). Gastrointest Endosc 2005; 62: 92-100.

7. Seifert H, Biermer M, Schmitt W, et al. Transluminal endoscopic necrosectomy after acute pancreatitis: a multicentre study with long-term follow-up (the GEPARD Study). Gut 2009; 58: 1260-66.

8. Papachristou GI, Takahashi N, Chahal P, et al. Peroral endoscopic drainage/debridement of walled-off pancreatic necrosis. Ann Surg 2007; 245: 943-51.

9. Gardner TB, Coelho-Prabhu N, Gordon SR, et al. Direct endoscopic necrosectomy for the treatment of walled-off pancreatic necrosis: results from a multicenter U.S. series. Gastrointest Endosc 2011; 73: 718-26.

10. Rische S, Riecken B, Degenkolb J, et al. Transmural endoscopic necrosectomy of infected pancreatic necroses and drainage of infected pseudocysts: a tailored approach. Scand I Gastroenterol 2013; 48: 231-40.

11. van Brunschot S, Fockens P, Bakker OJ, et al. Endoscopic transluminal necrosectomy in necrotising pancreatitis: a systematic review. Surg Endosc 2014; 28: 1425-38.

12. Jürgensen C, Neser F, Boese-Landgraf J, et al. Endoscopic ultrasound-guided endoscopic necrosectomy of the pancreas: is irrigation necessary? Surg Endosc 2012; 26: 1359-63.

Received: 30.12.2014, accepted: 5.03.2015. 